\title{
Rehabilitación sísmica de edificaciones históricas en tapia pisada: estudio de caso de capillas doctrineras reforzadas con malla de acero y madera de confinamiento
}

\section{Seismic rehabilitation of rammed-earth heritage buildings: study case of doctrinal churches reinforced with metallic mesh and confinement wooden elements}

V. Gómez ${ }^{(*)}$, C. López ${ }^{(*)}, \underline{\text { D. Ruiz }}{ }^{(*)}$

\section{RESUMEN}

A nivel latinoamericano, hay pocas investigaciones sobre iglesias (capillas) coloniales construidas en tapia pisada desde el punto de vista arquitectónico, histórico y estructural (desempeño sísmico). La carencia de estudios ha producido malas intervenciones del patrimonio histórico. En el presente artículo se presentan los resultados de ensayos en mesa vibratoria de modelos a escala 1:50 de capillas de adoctrinamiento en tapia pisada construidas en los siglos XVI al XvIII en Colombia. Los muros de los modelos de las capillas fueron rehabilitados sísmicamente mediante dos técnicas de reforzamiento diferentes: maderas de confinamiento y malla de acero; ambas técnicas instaladas por una sola cara de los muros. Los resultados sugieren que los sistemas de refuerzo sísmico evaluados disminuyen hasta en un 52 \% los desplazamientos de los muros a la vez que disminuyen la fisuración y el agrietamiento.

Palabras clave: Iglesias en tapia pisada; ensayos en mesa vibratoria; refuerzo estructural de edificaciones históricas; desempeño sísmico estructural; Colombia.

\section{ABSTRACT}

In Latin America, there is little research on colonial churches (chapels) built with earth (rammed earth) taking into account architectural, historical and structural (seismic performance) considerations. The lack of studies have produced bad interventions of the historical heritage. In this paper, the authors present results of shake table tests of 1:50 scale models of doctrinal rammed-earth chapels built in Colombia in the sixteenth and eighteenth centuries. The walls of the models of the chapels were seismically strengthened with two different techniques: confinement wooden elements and steel mesh; both techniques applied only on one side of the walls. The results suggest that the seismic reinforcement systems evaluated diminishes the displacement of the walls up to $52 \%$ and reduce the cracking.

Keywords: Rammed-earth churches; shake table tests; structural reinforcement of historic buildings; seismic structural performance; Colombia.

(*) Pontificia Universidad Javeriana. Bogotá, Colombia.

Persona de contacto/Corresponding author: daniel.ruiz@javeriana.edu.co (D. Ruiz)

Cómo citar este artículo/Citation: Gómez, V, López, C., Ruiz, D. (2016). Rehabilitación sísmica de edificaciones históricas en tapia pisada: estudio de caso de capillas doctrineras reforzadas con malla de acero y madera de confinamiento. Informes de la Construcción, 68(541): e140, doi: http://dx.doi.org/10.3989/ic.15.017.

Licencia / License: Salvo indicación contraria, todos los contenidos de la edición electrónica de Informes de la Construcción se distribuyen bajo una licencia de uso y distribución Creative Commons Reconocimiento no Comercial 3.o. España (cc-by-nc). 


\section{INTRODUCCIÓN Y JUSTIFICACIÓN}

El proceso de evangelización de la población indígena en Colombia, contribuyó a la fundación de nuevas poblaciones especialmente en el área central del país. Al llegar los españoles al área andina encontraron cerca de 300 caseríos de indios muiscas (1), en donde se establecieron doctrinas, que agrupaban los indios dominados para ser explotados como renta e instrumento de producción. El trazado del centro doctrinero, se comenzaba por la plaza alrededor de la cual se ubicaba la iglesia, el atrio, las capillas posas y la casa cural; luego se procedía al repartimiento de solares entre los conquistadores y caciques de cada tribu.

Las iglesias poseían una tipología llamada templos doctrineros (capillas o iglesias doctrineras) que se edificaban de acuerdo a lo establecido en las Leyes de Indias y los contratos de construcción. La construcción era costeada por los encomenderos y los indios contribuían con su trabajo y alimentos para el constructor. Los sacerdotes doctrineros tenían la misión de completar la conquista y fueron los primeros educadores en el nuevo continente. La iglesia era el eje formativo de los nuevos pueblos y servía como base para el desarrollo de lo urbano. (Figura 1).

En los contratos de construcción de las capillas doctrineras se definían aspectos arquitectónicos y estructurales. Algunas de las especificaciones ordenadas en los contratos eran (2):

- Ancho de la capilla doctrinera: entre 8,4 m y 10,1 m

- Longitud de la capilla doctrinera: 42 a $45 \mathrm{~m}$

- Altura de la capilla doctrinera: $5 \mathrm{~m}$

- Tipo de cubierta: Sistema de par y nudillo

- Capilla mayor: Debía construirse cuadrada u ochavada

- Iluminación: Debían construirse 10 ventanas, 6 para el cuerpo de la iglesia y 4 para el presbiterio

- Acabados de puertas y ventanas: Debían construirse en madera de acuerdo a los parámetros dados para iluminación.

Basados en esta tipología se han encontrado 41 iglesias (3) en el área andina colombiana, aunque el número de edificaciones construidas con este tipo de contrato fue de 125 . De estas han sido declaradas hasta la fecha como Monumentos Nacionales solo 22 iglesias (4).

\section{SISTEMA CONSTRUCTIVO}

De acuerdo visitas técnicas hechas a más de 10 capillas doctrineras, se presentan las principales características del sistema constructivo.

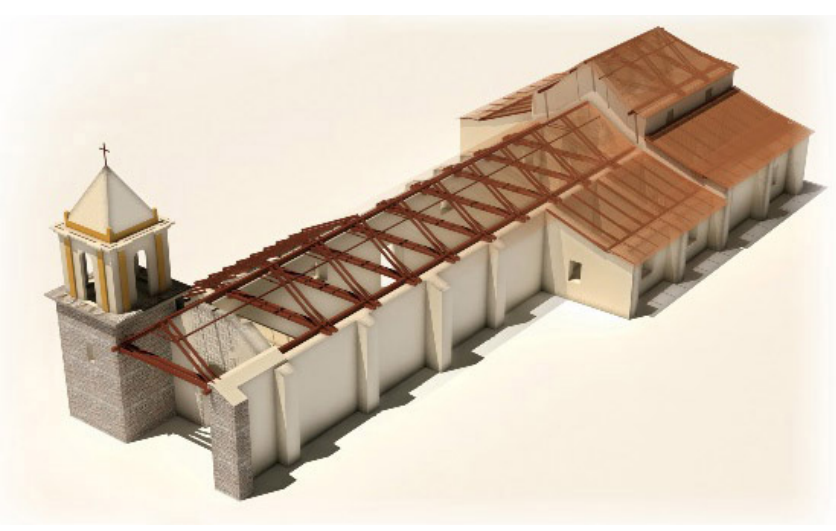

Figura 1. Volumetría de la Iglesia de Suesca en la zona Andina Colombiana.

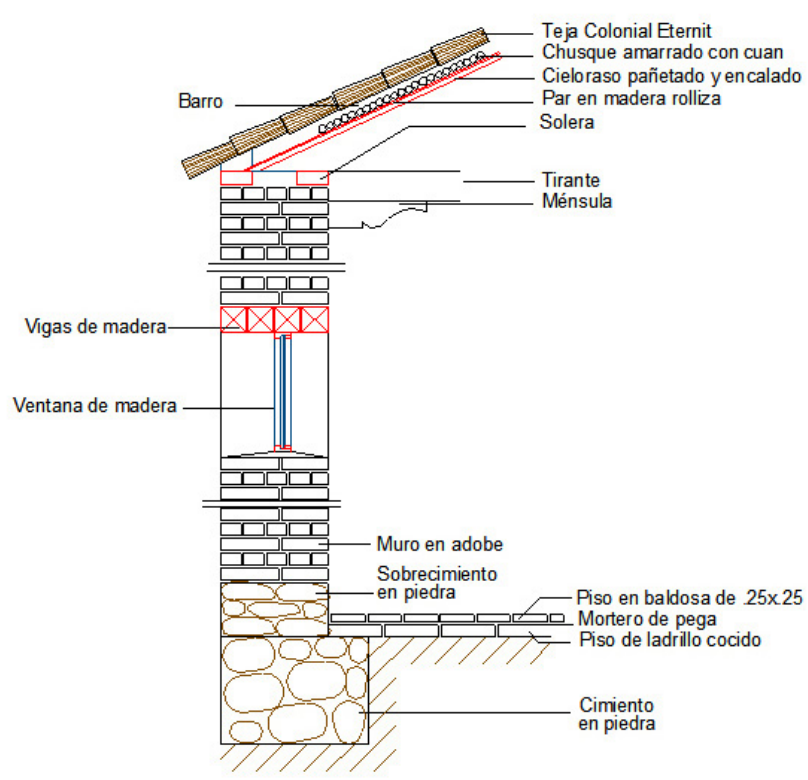

Figura 2. Muro típico de templo doctrinero.

\subsection{Cimentación y sobre-cimiento}

Los cimientos presentan una forma rectangular con una profundidad de 1,30 $\mathrm{m}$ y ancho hasta dos veces el espesor del muro (Figura 2). Para su construcción se emplearon piedras pegadas con una mezcla de barro y cal. El sobrecimiento encontrado tiene alturas promedio de $0,40 \mathrm{~m}$. Para proteger el muro de la humedad del suelo, se encontró evidencia de diferentes impermeabilizantes naturales como: brea, betún, aislantes naturales o piedras planas (lajas).

\subsection{Muros}

Se encontraron anchos de los muros hasta de 1,26 $\mathrm{m}$ y alturas entre 5,04 m y 6,72 m (Figura 2). La técnica constructiva predominante es la tapia pisada (Rammed earth). Los vanos de puertas y ventanas en el siglo XVI se realizaban en adobe y en la parte superior se colocaba un dintel en madera con un rebase de aproximadamente $0,3 \mathrm{~m}$ de cada lado del vano. Los pañetes se aplicaban como protección del muro empleando una mezcla de cal, productos aglutinantes y fibras naturales.

\subsection{Vigas de coronación y estructura de cubierta}

Las vigas de coronación se colocaban en la parte superior del muro y recibían la estructura de cubierta. Consistían en madera rolliza o escuadrada (entre $0,20 \mathrm{~m}$ y $0,30 \mathrm{~m}$ ); y se le cubrían con un impermeabilizante natural. El techo era a dos aguas con una inclinación entre 38 y 45 grados. La estructura de cubierta era una armadura triangular de par y nudillo en madera rolliza apoyada sobre las vigas de coronación. Sobre la cubierta se colocaba un entramado de chusque y cuan y una capa de barro. El acabado de cubierta se hacía con paja y posteriormente con tejas de barro.

\section{VULNERABILIDAD SÍSMICA DE CAPILLAS DOCTRINERAS EN TIERRA}

De acuerdo con (5) y (6), los principales problemas estructurales de las construcciones en tapia pisada son: poca cohesión estructural, erosión y desintegración. Adicionalmente el agua 


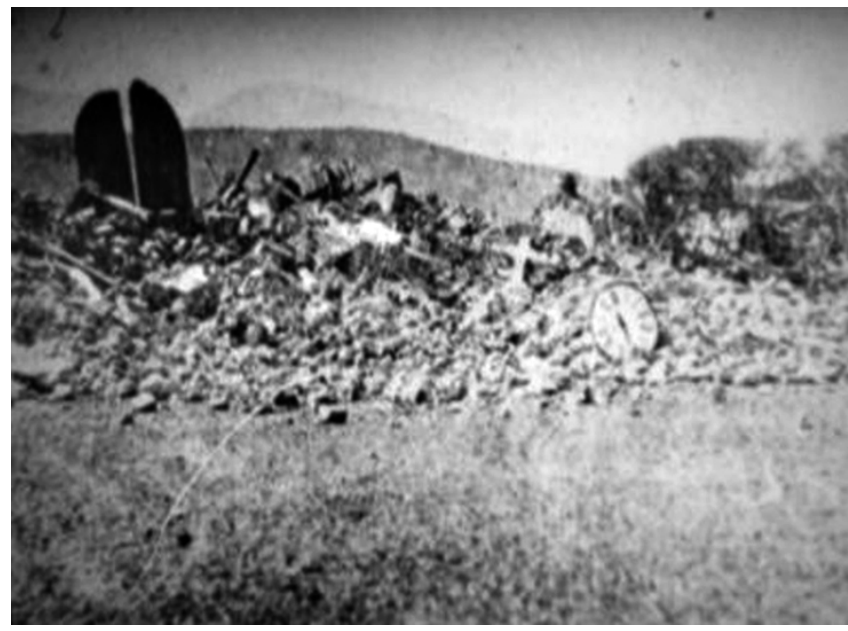

Figura 3. Colapso de la Iglesia de Cúcuta (en tierra) durante el terremoto de 1875 Tomado de (7).

puede generar problemas de humedad en los muros (pérdida de resistencia). Lo anterior trae como consecuencia una tendencia de las edificaciones en tierra a colapsar durante terremotos, como el sismo de Cúcuta (Colombia) en 1875 que causó el colapso de la iglesia (Figura 3).

Las capillas doctrineras en tierra presentadas en este artículo están localizadas en los Andes Colombianos en donde la amenaza sísmica es intermedia y alta. De acuerdo con (8), hay dos factores que originan el colapso de los muros hechos en tierra: a) el colapso de la cubierta, que afecta a los muros durante su caída; y b) las grietas de los muros, debido a la baja resistencia a esfuerzos de tensión y cortante. Esto ha sido comprobado por investigaciones experimentales y/o numéricas mostradas en (9), (10) y (11). De acuerdo con (9), la vulnerabilidad de las capillas doctrineras se incrementa al no poseer un diafragma rígido. Considerando los modelos por elementos finitos desarrollados por (9), los movimientos sísmicos (acordes con la amenaza sísmica de los Andes Colombianos) generarían esfuerzos a tracción de o,6 MPa, los cuales son mucho más grandes que la resistencia a tracción reportada para edificaciones construidas en tierra en Colombia que es de 0,02 MPa (7). Esto implica que las capillas doctrineras tienen un desempeño deficiente ante terremotos por lo se deben implementar alternativas de refuerzo.

\section{ALTERNATIVAS DE REHABILITACIÓN EVALUADAS}

En las últimas décadas se han propuesto diferentes alternativas de rehabilitación sísmica de edificaciones en tierra. Las técnicas de rehabilitación evaluadas en el presente estudio son: malla metálica con mortero de cal -presentadas en (8), (12) y (13) - y maderas de confinamiento -(8) y (14)-; ambas técnicas instaladas en ambas caras de los muros. La gran desventaja de estas dos técnicas se presenta cuando en los muros internos del monumento arquitectónico en tierra se tiene pintura mural o adornos, en cuyo caso no es posible instalar el refuerzo al interior de las capillas doctrineras. En los autores (14) y (8) se verifican las grandes ventajas estructurales de implementar el refuerzo por ambas caras de los muros, sin embargo no se estudió qué sucedía con el comportamiento estructural cuando se intervenía solamente una de las dos caras principales de los muros. Debe mencionarse que los restauradores en Colombia han usado malla con revestimiento plástico o con pintura anti- corrosiva, lo cual minimiza el efecto de la corrosión. A la escala a la que se hicieron los ensayos no se tomaron dichas precauciones ya que transcurrió poco tiempo desde que se instalaron los refuerzos hasta que se hicieron los ensayos.

A continuación se describirán las dos técnicas de refuerzo, con la salvedad que en los modelos de mesa vibratoria se usó el refuerzo por una sola cara de los muros.

\subsection{Reforzamiento con maderas de confinamiento}

El refuerzo propuesto por (8) y mostrado en la Figura 4 consiste en instalar elementos de madera (de $200 \mathrm{~mm}$ de ancho, $20 \mathrm{~mm}$ de espesor) en el plano del muro e interconectarlos mediante pernos pasantes. Se instalan maderas tanto verticales como horizontales espaciadas cada 1,5 metros; instalando maderas adicionales en las esquinas y en los vanos (puertas y ventanas). El sistema se complementa con uniones en las esquinas con ángulos de acero. Este reforzamiento incrementa la resistencia a flexión de los muros, mantiene la unidad de la estructura y da mayor capacidad de disipación de energía.

En el presente artículo se evalúa el comportamiento de los muros reforzados sólo por la cara externa. Por esta razón con el fin de que la cantidad de refuerzo (madera) por metro cuadrado de los muros se mantuviera, se decidió considerar tablas del doble de ancho de las recomendadas en (8).

\subsection{Reforzamiento con malla y mortero de cal}

Consiste en instalar una malla de vena por franjas horizontales y verticales (simulando vigas y columnas de confinamiento) en las zonas críticas de la estructura. La malla, se une a la pared mediante alambrones de $8 \mathrm{~mm}$ de diámetro espaciado cada $500 \mathrm{~mm}$. Posteriormente se recubre con mortero de cal y arena en proporción 1:2. Este refuerzo evita la pérdida de rigidez lateral que se presenta en edificaciones no reforzadas cuando se agrietan. En la Figura 5 se esquematizan los detalles de este tipo de rehabilitación que ha demostrado sus cualidades en eventos sísmicos (15). Esta técnica de rehabilitación permite incrementar la capacidad de disipación de energía de los muros ya que el sistema estructural alcanza mayores niveles de desplazamiento en el rango inelástico. Resultados similares, aunque con otro tipo de malla (mallas plásticas), fueron reportados por (16).

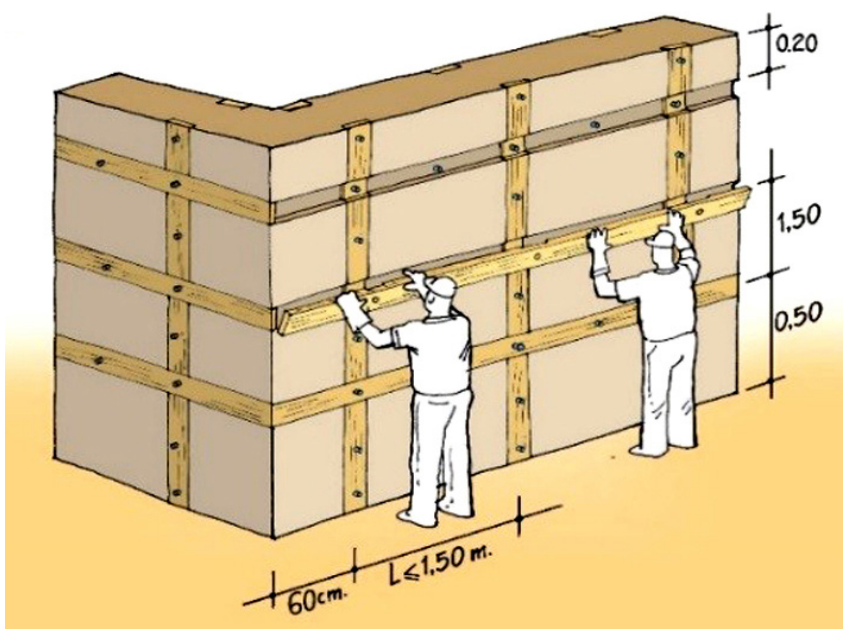

Figura 4. Proceso de rehabilitación de un muro en tierra con maderas de confinamiento (8). 


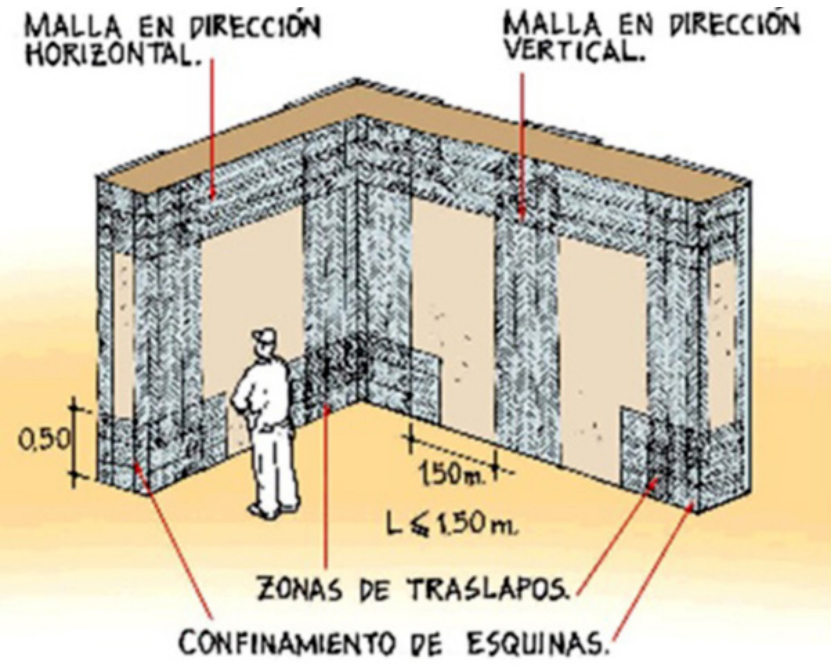

Figura 5. Proceso de instalación del refuerzo con malla metálica (8).

\section{FABRICACIÓN DE LOS MODELOS EXPERIMENTALES}

Se construyeron tres modelos a escala 1:50 de una capilla doctrinera para ser ensayados en la mesa vibratoria de la Pontificia Universidad Javeriana. El primer modelo se construyó sin ningún tipo de refuerzo, el segundo modelo se reforzó con malla y mortero y el tercer modelo se reforzó con maderas de confinamiento. El suelo usado para la construcción de los muros de las capillas doctrineras a escala fue el mismo usado en la referencia (14).

Las dimensiones de los diferentes muros que conforman los tres modelos (a escala) se presentan en la Tabla 1. Todos los muros tienen un espesor de $20 \mathrm{~mm}$, lo cual equivale a $1 \mathrm{~m}$ a escala real.

Para la construcción de los modelos se usaron formaletas metálicas con las que se construyó tanto los muros como la cimentación de los mismos. Se simuló la cimentación mediante la instalación de los muros a escala en tapia pisada sobre un canal de $20 \mathrm{~mm}$ de espesor y $20 \mathrm{~mm}$ de profundidad el cual se llenó con una mezcla de 2 partes de material granular (pasa tamiz $\mathrm{N}^{\circ} 4$ y retenido en Tamiz $\mathrm{N}^{\circ}$ 10.), 3 partes de arena, 1/2 partes de cal y agua. Posteriormente se ubicaron las láminas de la formaleta, amarrándola y ajustándola para garantizar el espesor de todos los muros aún después de la compactación del material. Luego se erigieron los muros, apisonando, con un compactador de madera de $400 \mathrm{~mm}^{2}$ de área transversal, por capas de $20 \mathrm{~mm}$ de altura. La mezcla usada para los muros fue de 2/3 partes de arena, por 1/9 partes de arcilla, 1/5

Tabla 1. Dimensiones a escala del modelo experimental.

\begin{tabular}{|l|c|c|c|}
\hline & $\begin{array}{c}\text { Altura } \\
\text { (mm) }\end{array}$ & $\begin{array}{c}\text { Longitud } \\
\text { (mm) }\end{array}$ & $\begin{array}{c}\text { Ancho } \\
\text { (mm) }\end{array}$ \\
\hline Torre & 183 & 78 & 78 \\
\hline Baptisterio & 83 & 142 & 128 \\
\hline Nave Central & 120 & 762 & 160 \\
\hline Presbiterio & 160 & 252 & 160 \\
\hline Capilla Lateral Izquierda & 100 & 252 & 125 \\
\hline Capilla Lateral Derecha & 100 & 252 & 114 \\
\hline Sacristía & 100 & 128 & 114 \\
\hline Depósito & 100 & 130 & 114 \\
\hline
\end{tabular}

partes de cal apagada y fibra de fique. Durante éste proceso se colocaron puertas y ventanas. A continuación se instaló la viga de coronación en madera; y durante un mes los modelos se secaron lentamente con el fin de evitar la aparición de fisuras. Una vez hecho esto a cada modelo se le instaló la cubierta en madera que soportaría las masas sobre impuestas del modelo dinámico. La masa de las edificaciones en tierra proviene en gran medida de los muros (por su gran espesor) y alta densidad.

Adicional a los muros, la masa que aporta a la fuerza inercial es la de la cubierta, la cual está compuesta por una capa de tierra, tejas de barro, fibras vegetales y armaduras de madera. Como la escala geométrica fue de 1:50; la escala de la masa de los muros (volumen) fue de 1:(50)3 (1:125000). Para la masa de la cubierta se mantuvo esta escala de masa. De acuerdo con lo anterior, el peso de la cubierta de la capilla doctrinera a escala real es de $13200 \mathrm{kN}\left(17 \mathrm{kN} / \mathrm{m}^{2}\right)$, de tal manera que el peso para el modelo a escala en la cubierta fue de 105 N. Con base en lo anterior los autores ubicaron sobre la cubierta 20 pesas de 5,o N. En la Figura 6 se muestra una fotografía del modelo sin refuerzo instalado sobre la mesa vibratoria y con la instrumentación, la cubierta y las masas instaladas.

El refuerzo en madera a escala consistió en chapilla de cedro, usando un entramado de listones de $8 \mathrm{~mm}$ de ancho, separados cada $30 \mathrm{~mm}$ entre ejes. En las esquinas de los muros el refuerzo se unió con lámina de latón calibre 20, y en los traslapos de los listones horizontales y verticales se pasó un alambre de cobre y se selló con soldadura de estaño. Debe recordarse que a diferencia de lo presentado en (14) este modelo fue reforzado por una sola cara (la cara externa).

Se presenta en la Tabla 2 una comparación entre el refuerzo recomendado a escala real propuesto en (8) y (14), su equivalente a escala 1:50 y el refuerzo implementado en los modelos del presente artículo. Para mantener la misma cantidad de

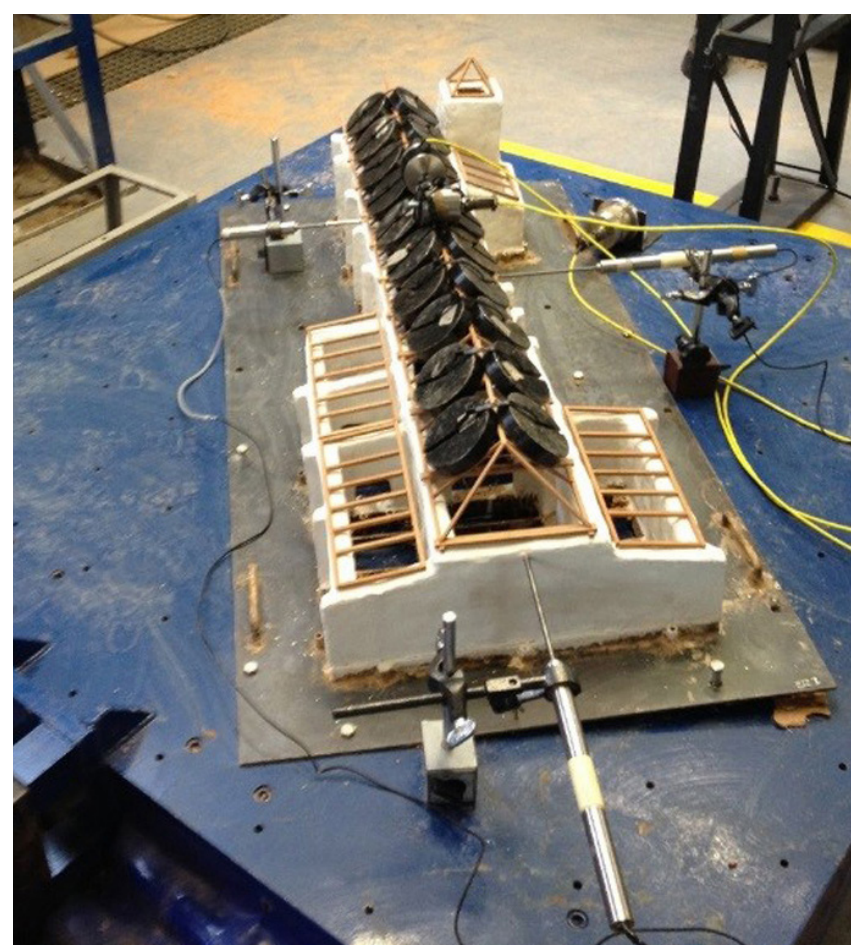

Figura 6. Capilla doctrinera a escala sin refuerzo (instalada en la mesa vibratoria) con la instrumentación para las pruebas. 
Tabla 2. Dimensiones de los refuerzos en madera y malla implementados en los modelos de mesa vibratoria.

\begin{tabular}{|l|c|c|c|}
\hline \multirow{2}{*}{\multicolumn{1}{|c|}{ Ítem }} & \multicolumn{2}{c|}{$\begin{array}{c}\text { Refuerzo recomendado por las } \\
\text { referencias (8) y (14) en muros } \\
\text { reforzados por las dos caras }\end{array}$} & $\begin{array}{c}\text { Refuerzo propuesto en el presente } \\
\text { documento para muros reforzados } \\
\text { por una sola cara }\end{array}$ \\
\cline { 2 - 5 } & Escala real & Escala 1:50 & Escala real \\
\hline Ancho de las tablas de madera (mm) & 200 & 4,0 & 400 \\
\hline Espesor de las tablas de madera (mm) & 20 & 0,40 & 22 \\
\hline $\begin{array}{l}\text { Separación (entre ejes) de las tablas de } \\
\text { madera (mm) }\end{array}$ & 1500 & 30 & 1500 \\
\hline Ancho de las franjas de malla (mm) & 500 & 10 & 30 \\
\hline $\begin{array}{l}\text { Separación entre bordes externos de las } \\
\text { franjas de malla (mm) }\end{array}$ & 1500 & 30 & 300 \\
\hline
\end{tabular}

refuerzo por $\mathrm{m}^{2}$ en los modelos que serían reforzados por una sola cara, se usaron maderas del doble de ancho de lo recomendado por (8) (en vez de $4 \mathrm{~mm} 8 \mathrm{~mm}$ ). Para el caso del refuerzo con malla a escala, la malla instalada tenía el doble de la densidad por unidad de área de la malla a escala real. Por esta razón se mantuvo el ancho de las franjas verticales y horizontales en $10 \mathrm{~mm}-500 \mathrm{~mm}$ a escala real recomendado por la referencia (8)-. Esto teniendo en cuenta la directriz de la investigación de tratar de poner en una sola cara de los muros la totalidad del refuerzo recomendado para dos caras.

En la Figura 7 se muestra el modelo con refuerzo en maderas de confinamiento por una sola cara.

En la Figura 8 se muestra la técnica que usa malla de acero y mortero de cal. La malla se instala conformando franjas ver-

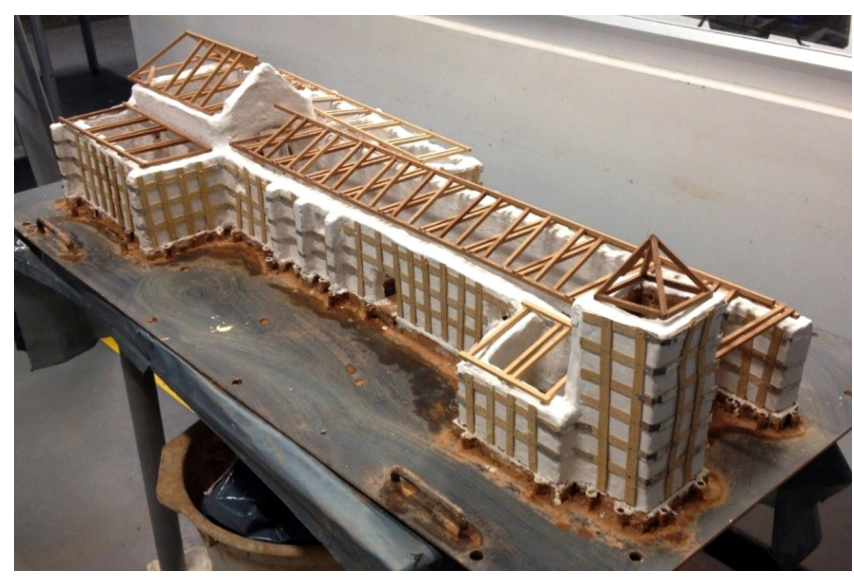

Figura 7. Capilla doctrinera con refuerzo de maderas de confinamiento por la cara externa.

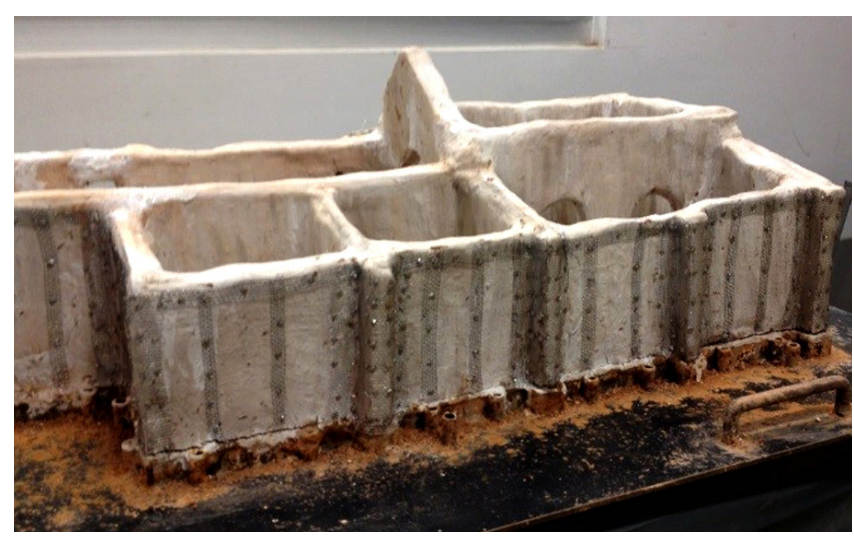

Figura 8. Proceso de instalación del refuerzo con malla y mortero en las capillas a escala. ticales y horizontales. Los modelos numéricos y la evidencia encontrada en campo, demuestran que las uniones entre los muros y los contrafuertes (elementos de soporte perpendicular al plano del muro) son puntos críticos ya que cuando estas conexiones se fracturan el muro aumenta su longitud no arriostrada induciendo fallas por flexo-tracción. Por esta razón se deben reforzar todas las uniones contrafuerte-muro mediante la instalación de una malla que envuelve al contrafuerte. Con base en lo anterior en la Figura 8 se aprecian las bandas verticales y las horizontales y los contrafuertes forrados con malla. Una vez se instala la malla con anclajes se aplica mortero de cal y arena.

Los tres modelos se instalaron en la mesa vibratoria a un ángulo de 45 grados con respecto a la dirección principal de movimiento para generar fuerzas de flexión y de cortante en los muros. Se ubicaron masas en la cubierta, distribuidas en las cerchas de la misma. Es importante mencionar que de acuerdo con la referencia (17), para que los resultados sean extrapolables a modelos reales de capillas doctrineras; tendría que adosarse una cantidad de masa tal que equivaldría a instalar un muro de plomo a cada muro de tierra con sus mismas dimensiones; lo cual es imposible. Sin embargo en la misma referencia se abre la posibilidad de desarrollar modelos semi-cualitativos en donde es posible modificar la escala de tiempo de la señal de movimientos del terreno para incrementar artificialmente la aceleración. Al seguir este procedimiento alternativo, los resultados entre los modelos con y sin refuerzos pueden ser comparados entre sí; siendo esta la alternativa seguida para la presente investigación y que ya fue implementado exitosamente en (14) y (8).

Los tres modelos fueron instrumentados con tres acelerómetros sísmicos, uno en dirección longitudinal al modelo, otro en dirección transversal y un tercero instalado en dirección del movimiento de la mesa vibratoria. Tres de los muros principales fueron instrumentados con deformímetros tipo LVDT; de tal manera que se registró el movimiento relativo de los dos muros principales de la nave central y el muro exterior que comparten el presbiterio y las capillas laterales izquierda y derecha.

\section{RESULTADOS DE LOS ENSAYOS EN MESA VIBRATORIA}

Los ensayos se realizaron en la mesa vibratoria del Laboratorio de Pruebas y Ensayos de la Pontificia Universidad Javeriana. La mesa uniaxial es accionada por un actuador dinámico MTS de $100 \mathrm{kN}$ de capacidad de carga que tiene un recorrido total de $250 \mathrm{~mm}(+/-125 \mathrm{~mm})$. La plataforma de la mesa vibratoria es cuadrada con 1,5 m de lado y el conjunto 
actuador-mesa puede generar aceleraciones hasta de 5,o g en modelos estructurales. La señal sísmica usada es acorde a la amenaza sísmica de Bogotá, capital de Colombia y corresponde a un sismo de origen regional, con epicentro a $40 \mathrm{~km}$ en la Capital y magnitud de 7,6 Mw (aceleración máxima en roca de 0,2 g) (ver Figura 9). Esta señal ha sido usada anteriormente en pruebas sobre modelos a escala en tierra en Colombia tal como lo reportado en (8) y (14). La señal fue modificada de acuerdo con lo establecido por (17) para modelos a escala, de tal manera que la señal sísmica, que generó movimientos a los modelos, tuvo una duración menor pero con un mayor nivel de aceleración.

En las Figuras 10, 11 y 12 se presentan imágenes del estado final de los modelos experimentales de las capillas doctrineras a escala después de los ensayos en mesa vibratoria. Es evidente en la Figura 10 que el sismo generó colapsos de muros en la capilla doctrinera sin ningún tipo de refuerzo con caída de los muros en las capillas laterales. Estos resultados y la evidente fragilidad son consistentes con lo encontrado en la literatura y con la pobre resistencia a la tensión y el cortante de la mampostería de tierra. Los mecanismos de colapso y de falla de la capilla sin refuerzo son típicos de los muros de tierra según lo reportado por (8); debidos a la baja resistencia a esfuerzos cortantes y la nula resistencia a esfuerzos de tracción.

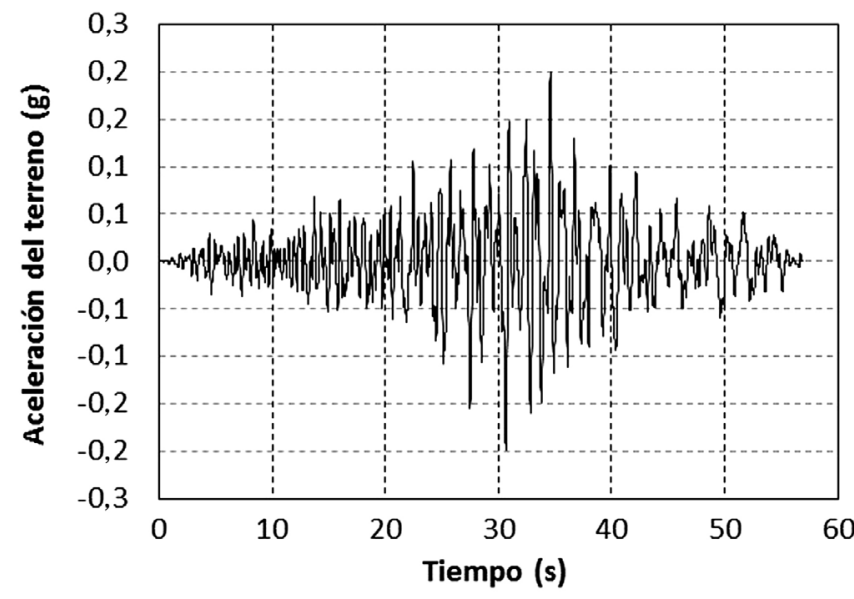

Figura 9. Señal sísmica representativa de la amenaza sísmica de Bogotá (Sismo Regional).

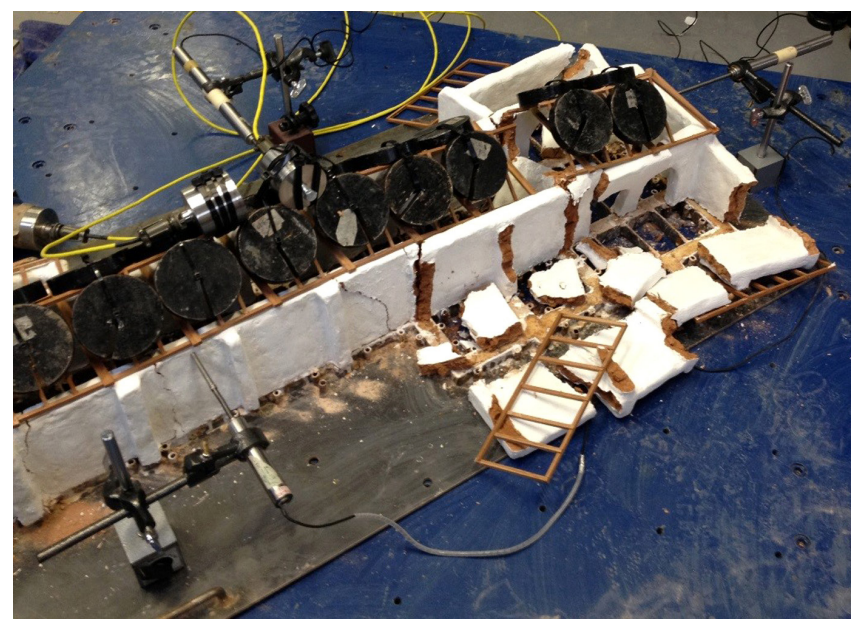

Figura 10. Colapso de una de las secciones de la capilla doctrinera sin refuerzo.
Por su parte en los modelos reforzados no se presentó la caída de ninguno de los muros y la estabilidad de los muros se garantizó aunque se presentaron fisuras y grietas como se muestra en las Figuras 11 y 12.

En el modelo reforzado con malla metálica la integridad estructural se mantiene y la nave central en general no presenta mayor afectación. En total aparecieron sólo seis fisuras de alguna importancia en todo el modelo y no hubo colapsos. En la zona lateral izquierda aparece una fisura vertical entre contrafuertes, atravesando un pequeño vano de ventana y en la zona frontal; Asimismo el arco de acceso principal presenta fisuras verticales. Se presentó una fisura entre la capilla lateral derecha y el muro posterior debido a los esfuerzos inducidos en la unión de los muros. En un muro interior de la capilla lateral derecha se presenta una fisura sobre dos de los arcos y una grieta en diagonal debida a esfuerzos cortantes (ver Figura 11).

Por su parte, en el modelo reforzado con maderas de confinamiento las fisuras fueron considerablemente menores a las presentadas en el ensayo del modelo reforzado con malla. Este mejor comportamiento ya fue reportado por (8). En general en este modelo con maderas no se observa ninguna fisura en el arco de acceso principal en la zona frontal y en toda la capilla doctrinera aparecieron tres fisuras. Sobre la zona

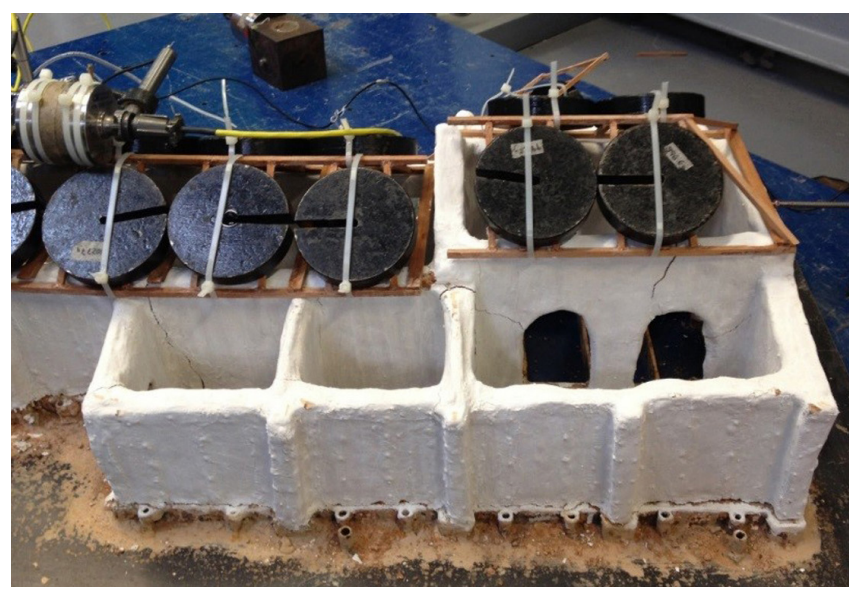

Figura 11. Grietas después del ensayo en la capilla doctrinera reforzada con malla y mortero.

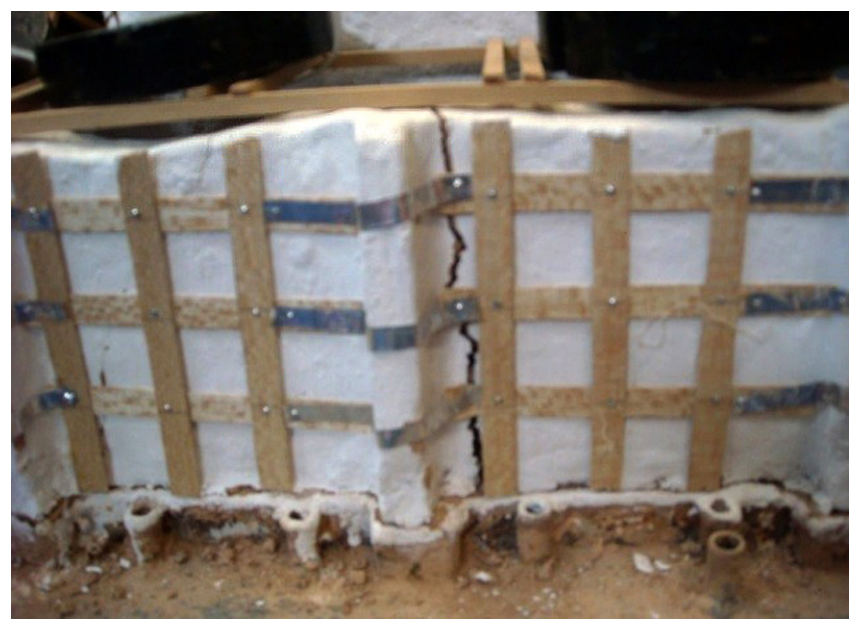

Figura 12. Grieta generada en la capilla doctrinera reforzada con madera de confinamiento. 


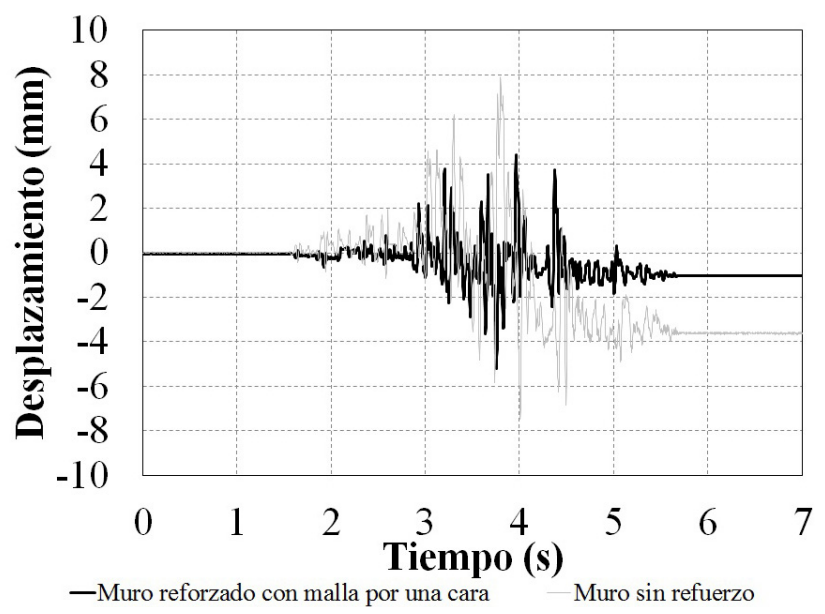

Figura 13. Desplazamientos del muro de la nave central de la capilla doctrinera reforzada con malla y mortero.

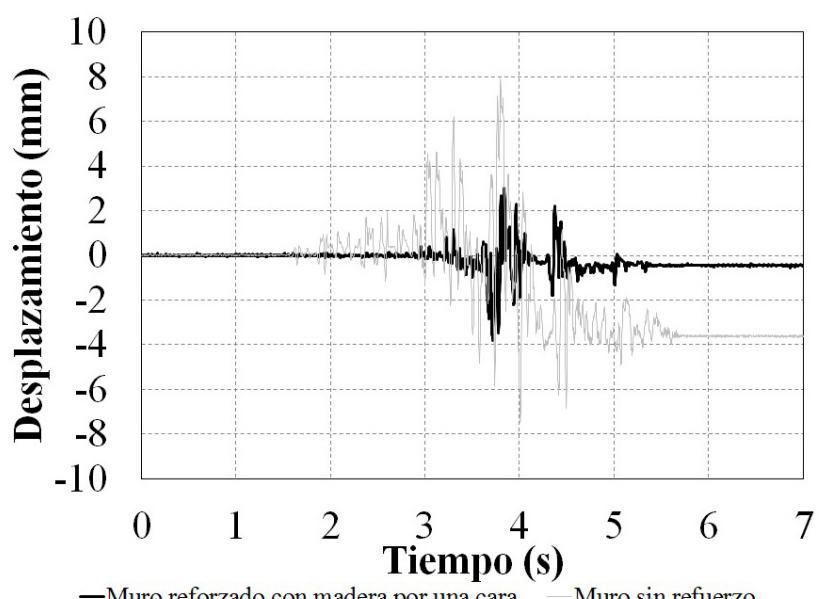

Figura 14. Desplazamientos del muro de la nave central de la capilla doctrinera reforzada con maderas de confinamiento.

lateral izquierda se observó una fisura vertical en la capilla y el baptisterio y hay también una fisura vertical en uno de los arcos del presbiterio. En la capilla lateral derecha aparece una grieta vertical junto al contrafuerte, pero se mantiene la integridad como se aprecia en la Figura 12.

El anterior análisis cualitativo fue corroborado por las mediciones registradas con los deformímetros tipo LVDT. En las Figuras 13 y 14 se presentan los desplazamientos relativos (entre la mesa vibratoria y la estructura) registrados en el muro más largo de la nave central para la condición sin y con refuerzo. Los desplazamientos máximos del muro sin refuerzo llegaron hasta $7,9 \mathrm{~mm}$, mientras que los desplazamientos en el mismo muro, pero reforzado con malla y madera, fueron menores con valores de $5,2 \mathrm{~mm}$ y $3,8 \mathrm{~mm}$ respectivamente.
Por su parte los registros de las Figuras 13 y 14 evidencian que al final del ensayo el muro tuvo desplazamientos permanentes residuales como consecuencia del daño generado por el movimiento sísmico. El muro sin refuerzo tuvo un desplazamiento permanente de $3,7 \mathrm{~mm}$ y el mismo muro pero reforzado con malla y madera tuvo un desplazamiento residual de $1,01 \mathrm{~mm}$ y $0,42 \mathrm{~mm}$ respectivamente. Es importante anotar que de acuerdo con lo reportado por (14), los muros reforzados con madera por ambas caras no presentan desplazamientos permanentes al finalizar el ensayo.

\section{CONCLUSIONES}

Teniendo como marco de referencia el diseño específico empleado en los ensayos y en los sistemas de reforzamiento, se presentan a continuación las conclusiones del trabajo:

El colapso de muros de tierra sólo se presentó en el modelo de la capilla doctrinera sin refuerzo, debido a: a) agrietamientos por concentración de esfuerzos en las esquinas y vanos; b) baja resistencia a corte y a tracción y c) baja capacidad de disipación de energía.

Los modelos de capillas doctrineras en tierra reforzados con malla metálica y madera evidenciaron un mejor desempeño sísmico que el modelo sin refuerzo. Esto porque disminuyeron los desplazamientos de los muros, los desplazamientos permanentes y hubo una menor aparición y propagación de fisuras.

De los dos esquemas de refuerzo analizados, las maderas de confinamiento tuvieron un mejor comportamiento, ya que: los muros reforzados con maderas presentaron menos fisuras y grietas después de los ensayos de mesa vibratoria; el desplazamiento máximo de los muros reforzados es un $48 \%$ del desplazamiento de los muros sin refuerzo y el desplazamiento permanente de los muros disminuyó en un $89 \%$. Los resultados experimentales sugieren que es posible realizar la rehabilitación sísmica estructural de muros de tapia pisada de capillas doctrineras en tierra solamente por la cara externa aunque los muros reforzados por una sola cara presentan mayor nivel de daño que los reforzados por ambas caras.

\section{AGRADECIMIENTOS}

La investigación presentada en este artículo fue financiada por el Departamento de Ingeniería Civil, el Departamento de Arquitectura y la Vicerrectoría de Investigación de la Pontificia Universidad Javeriana. Todos los ensayos fueron realizados en el Laboratorio de Pruebas y Ensayos del Departamento de Ingeniería Civil de la misma Universidad. El proyecto fue desarrollado por los grupos de investigación: Estructuras y Construcción; GRIME.

\section{REFERENCIAS}

(1) Corradine, A. (1989). Historia de la arquitectura colombiana. Colonia 1538-1850 (1ª Edición). Bogotá: Biblioteca Cundinamarca.

(2) Visitas Cundinamarca. Actas de visitas del oidor Luis Henríquez. (1601). Archivo General de la Nación (AGN), Sección 1. Colonia. Legajo: 5, Folios 746 a 903.

(3) López, C., Ruiz, D., Muñoz, E., Uribe, J., Maldonado, P., Jerez, S. (2005). Rehabilitación de arquitectura en tierra en el área andina. En Sismo Adobe 2005. Lima: Pontificia Universidad Católica del Perú.

(4) Colcultura. (1995). Catálogo Monumentos Nacionales de Colombia. Época colonial. Colombia. 
(5) Tavares, A., Costa, A., Varum, H. (2012). Common pathologies in composite adobe and reinforced concrete constructions. Journal Performance of Constructed Facilities, 26(4): 389-401, doi: http://dx.doi.org/10.1061/(ASCE)CF.19435509.0000200.

(6) Day, R. (1993). Performance of historic adobe structure. Journal Performance of Constructed Facilities, 7(3): 164-169, doi: http://dx.doi.org/10.1061/(ASCE)o887-3828(1993)7:3(164).

(7) Ruiz, D., López, C., Rivera, J. (2012). Propuesta de normativa para la rehabilitación sísmica de edificaciones patrimoniales. Revista Apuntes, 25(2): 226-239.

(8) Yamín, L., Phillips, C., Reyes, J., Ruiz, D. (2007). Estudios de vulnerabilidad sísmica, rehabilitación y refuerzo de casas en adobe y tapia pisada. Revista Apuntes, 20(2): 286-303.

(9) Ojeda, M., Muñoz, E. (2002). Análisis estructural de inmuebles patrimoniales en tierra y su comportamiento ante un sismo (Tesis de Maestría). Bogotá: Pontificia Universidad Javeriana.

(10) Rivera, J. C., Muñoz, E. E. (2005). Caracterización estructural de materiales de sistemas constructivos: Adobe. Revista Internacional de Desastres Naturales, Accidentes e Infraestructura Civil, 5(2): 135-148.

(11) López, C., Ruiz, D., Jerez, S., Quiroga, P., Uribe, J. (2007). Rehabilitación sísmica de muros de adobe de edificaciones monumentales mediante tensores de acero. Revista Apuntes, 20(2): 304-317.

(12) Blondet, M., Garcia, M., Gladys, V., Brzev, S. (2003). Earthquake-resistant construction of adobe buildings: A tutorial. Oakland, CA.: EERI/IAEE World Housing Encyclopedia.

(13) Blondet, M., Vargas, J., Tarque, N. (2008). Observed behavior of earthen structures during the Pisco (Perú) earthquake of August 15, 2007. En Proceedings of 14th World Conference on Earthquake Engineering. International Association for Earthquake Engineering (IAEE), Tokyo, Japan.

(14) Ruiz, D., López, C., Unigarro, S., Domínguez, M. (2014). Seismic Rehabilitation of Sixteenth- and Seventeenth-Century Rammed Earth-Built Churches in the Andean Highlands: Field and Laboratory Study. Journal of Performance of Constructed Facilities, 29(6): 04014144-1 - 04014144-17, doi: http://dx.doi.org/10.1061/(ASCE)CF.1943-5509.0000605.

(15) Blondet, M., Vargas, J., Tarque, N., Iwaki, C. (2011). Construcción sismorresistente en tierra: la gran experiencia contemporánea de la Pontificia Universidad Católica del Perú. Informes de la Construcción, 63(523): 41-50, doi: http:// dx.doi.org/10.3989/ic.10.017.

(16) Varum, H., Figueiredo, A., Silveira, D., Martins, T., Costa, A. (2011). Investigaciones realizadas en la Universidad de Aveiro sobre caracterización mecánica de las construcciones existentes en adobe en Portugal y propuestas de rehabilitación y refuerzo. Resultados alcanzados. Informes de la Construcción, 63(523):127-142, http://dx.doi.org/10.3989/ ic.10.016.

(17) Harris, H; Sabnis, G. (1999). Structural modeling and experimental techniques. Washington D.C.: CRC Press LLC. Doi: http://dx.doi.org/10.1201/9781420049589. 